\title{
SISTEMA DE POSICIONAMIENTO EN INTERIORES BASADO EN SEÑAL ÓPTICA. CALIBRACIÓN RÁPIDA
}

\author{
David Rodríguez \\ Departamento de Electrónica. Escuela Politécnica Superior. Universidad de Alcalá. david.rodriguez@uah.es
}

Álvaro de la Llana

Departamento de Electrónica. Escuela Politécnica Superior. Universidad de Alcalá. alvaro.llana@uah.es

José Luis Lázaro

Departamento de Electrónica. Escuela Politécnica Superior. Universidad de Alcalá. josel.lazaro@uah.es

Alfredo Gardel

Departamento de Electrónica. Escuela Politécnica Superior. Universidad de Alcalá. alfredo.gardel@uah.es

Felipe Espinosa

Departamento de Electrónica. Escuela Politécnica Superior. Universidad de Alcalá. felipe.espinosa@uah.es

Ignacio Bravo

Departamento de Electrónica. Escuela Politécnica Superior. Universidad de Alcalá. ignacio.bravo@uah.es

\section{Resumen}

Para obtener medidas precisas con sistemas de posicionamiento en interiores, por lo general, se requiere de una calibración de los mismos. En el caso de incorporar ópticas la calibración pueden requerir mucho tiempo, además de una infraestructura especializada. En este trabajo se presenta un método de calibración basado en Direct Linear Transformation, (DLT), que obtiene directamente la transformación entre el sensor y la superficie de movimiento del agente, que proporciona resultados comparables a una calibración óptima, siendo las diferencias de determinación de la posición de tan solo algunos milimetros.

Palabras clave: sistema de posicionamiento en interiores, sensor de posición óptico, red de sensado inalámbrico, robótica

\section{INTRODUCCIÓN}

En la actualidad existen diversas tecnologías para el desarrollo de sistemas de posicionamiento en interiores con características que distinguen a cada uno de ellos. A diferencia de los sistemas globales de posicionamiento en exteriores, como es el GPS, los sistemas de posicionamiento en interiores suelen estar más personalizados a las aplicaciones. Las características a tener en cuenta para un sistema de posicionamiento en interiores son: Coste, precisión, cobertura, privacidad, velocidad de actualización de posición, consumo, mantenimiento, infraestructura, sistemas activos o pasivos, etc. Este artículo se centra en sistemas de posicionamiento en interiores basados en señales ópticas.

Tradicionalmente este tipo de sistemas se han dedicado a la medición de distancias grandes (Lidar), pero en los últimos años, y con el uso de dispositivos LED para iluminación, se están proponiendo sistemas para posicionamiento en interiores basados en VLC (Visible Light Communication) [1], tanto utilizando fotodiodos [2] o cámaras [3]. En [2] utilizan un solo led y un sensor inertial para determinar la posición usando AoA con precisiones de $29.8 \mathrm{~cm}$ en coberturas de $5 \times 1 \times 1.5$ metros.

Otro trabajo en esa línea se presenta en es [4], en el que basándose en RSS (Received Signal Strength) y 3 leds determinan la posición consiguiendo precisiones de pocos centímetros.

En lo referente al uso de cámaras, por ejemplo, se encuentran trabajos como [3] que utilizan una cámara y 3 leds de iluminación, ubicados en el entorno en posiciones conocidas, para obtener la relación entre los puntos en la cámara y el entorno utilizando métodos iterativos y conocen la posición en relación a la posición de los LEDs. Otro trabajo similar, aunque utilizando leds infrarrojos ubicados en el agente móvil y cámaras en el entorno, es [5].

El uso de esta tecnología se concentra en posicionamiento de agentes móviles. En nuestro caso, hemos desarrollado un receptor basado en sensores PSD (Position Sensitive Device) utilizando AoA 
(Angle of Arrival) como técnica de posicionamiento, consiguiendo precisiones menores a $1 \mathrm{~cm}$, velocidades de actualización de posición elevadas, baja carga computacional y receptor con posibilidad de comunicarse vía WIFI.

Un handicap del sistema desarrollado surge en aquellas aplicaciones donde el número de receptores a desplegar por el entorno es elevado, ya que cada uno de los receptores necesita de una calibración que, aunque es off-line, requiere de tiempo e infraestructura para llevarse a cabo. Dicha calibración sirve para la obtención de los parámetros que modelan el receptor y se divide en dos fases: Calibración eléctrica descrita en [6], y calibración geométrica desarrollada en [6].

En este artículo se propone un método para obtener los parámetros del modelo del sistema de posicionamiento que evite la parte de calibración "óptima" (tradicional) del sistema receptor, de manera que el despliegue de sensores en un entorno sea sencillo.

PROPUESTA DEL MODELO DEL SISTEMA

\subsection{DESCRIPCIÓN DEL SISTEMA RECEPTOR}

El receptor se compone de un sensor PSD [8] que es un fotodiodo con 4 ánodos y un cátodo común, que cuando impacta un haz de luz sobre su superficie genere una corriente que se divide entre sus ánodos de forma inversamente proporcional a las distancias entre el punto de impacto y la posición de los ánodos.

Con el nivel de señal de los ánodos se puede hallar la coordenada donde ha impactado el haz de luz sobre el área activa del sensor PSD con respecto a su centro, según las ecuaciones ideales (1) y (2) proporcionadas por los fabricantes.

$$
\begin{aligned}
& X=\frac{L_{x}}{2} \frac{\left(I_{1}+I_{2}\right)-\left(I_{3}+I_{4}\right)}{I_{1}+I_{2}+I_{3}+I_{4}} \\
& Y=\frac{L_{y}}{2} \frac{\left(I_{1}+I_{4}\right)-\left(I_{2}+I_{3}\right)}{I_{1}+I_{2}+I_{3}+I_{4}}
\end{aligned}
$$

Para hacer converger un haz de luz sobre el sensor se ha acoplado una lente, quedando el sistema receptor como se muestra en la figura 1 , siendo $X, Y$ las coordenadas del impacto, $I x$ las corrientes por los diferentes ánodos y $L_{x}, L_{y}$ las dimensiones del PSD.

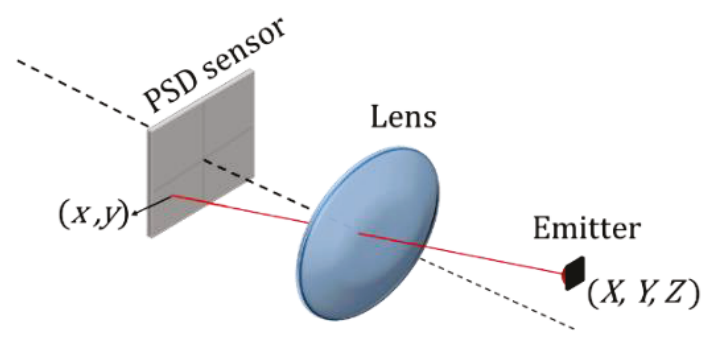

Figura 1: Representación del sistema receptor

\subsection{MODELO TEÓRICO}

En esta sección se describe es un método que permite determinar los parámetros del modelo del sensor de una forma sencilla, y a partir de esto determinar la posición de un agente móvil en el plano de movimiento.

La propuesta y procedimientos son los siguientes: en primer lugar, se coloca el sensor en el entorno sin ningún tipo de calibración previa. Dado que se asume que el sensor va a tener una cobertura fija sobre una superficie plana en la que se va a mover el emisor, se va a obtener directamente la transformación entre los puntos del plano y sus correspondientes en el sensor. Por tanto, para realizar medidas de posición posteriores, una vez obtenido un punto en el sensor se utilizará dicha transformación para obtener la posición correspondiente en el plano de movimiento. Este método de medida es menos preciso que una calibración óptima [9], pero también mucho menos costoso en términos de tiempo y de infraestructura necesaria, y sin riesgos de modificación de parámetros en el manipulado del sensor en su colocación.

En una primera aproximación se va a considerar un sistema lineal, y posteriormente se va a introducir la corrección de posibles distorsiones del sistema (tanto de lente como de PSD). Inicialmente no se plantea la corrección de distorsiones tangenciales (consideramos que no aportarán errores significativos), que harían más complejo el procedimiento.

Si se parte del esquema y modelo que se representa en la figura 2 , donde $\pi$ representa el plano de movimiento del emisor y $\mathrm{P}$ el plano imagen del receptor, si se conoce la relación de transformación $\boldsymbol{M}$, la posición del emisor en el plano $\pi$, a partir de un punto en el sensor, vendrá dada por (3):

$$
\left[\begin{array}{c}
s X \\
s Y \\
s
\end{array}\right]=M\left[\begin{array}{l}
x \\
y \\
1
\end{array}\right]
$$

donde $(x, y)$ representa un punto en el sensor PSD, $(X, Y)$ sus coordenadas en el plano de desplazamiento, 
y $\boldsymbol{M}$ es una matriz $3 \times 3$ que hace que el sistema matricial (3) quede como se muestra en (4).

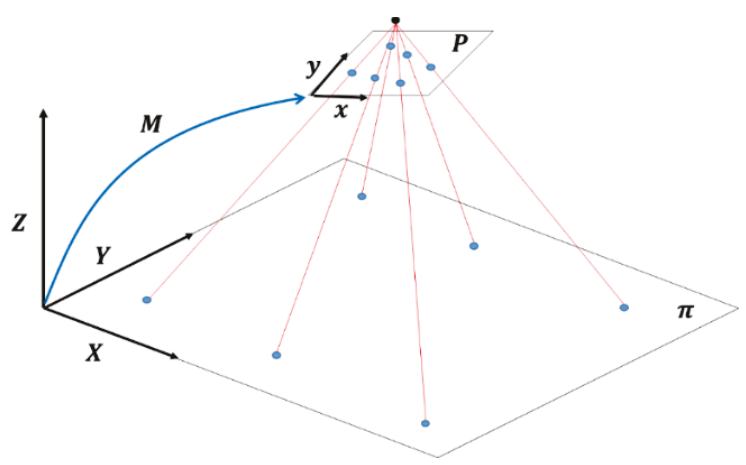

Figura 2: Relación entre el plano $\pi$ y el plano $P$

$$
\left[\begin{array}{c}
s X \\
s Y \\
s
\end{array}\right]=\left[\begin{array}{lll}
m_{11} & m_{12} & m_{13} \\
m_{21} & m_{22} & m_{23} \\
m_{31} & m_{32} & m_{33}
\end{array}\right]\left[\begin{array}{l}
x \\
y \\
1
\end{array}\right]
$$

\section{MÉTODO DE CALIBRACIÓN}

La calibración de la correspondencia entre los planos $\boldsymbol{\pi}$ y $\boldsymbol{P}$ o lo que es lo mismo la obtención de los parámetros $m_{i j}$ de la matriz $\boldsymbol{M}$ se realiza colocando el emisor en puntos conocidos del plano $\pi$ y captando dónde impactan en el sensor. La obtención de los parámetros, se realiza por medio de SVD (SingularValue Decomposition) a partir del sistema matricial (5), siendo necesarios 5 o más puntos para poder resolver el sistema. No obstante, es recomendable sobredimensionar el sistema y distribuir los puntos en el plano cubriendo la mayor parte posible del área de cobertura del sensor.

$$
\left[\begin{array}{lllllllll}
\boldsymbol{x} & \boldsymbol{y} & \mathbf{1} & \mathbf{0} & \mathbf{0} & \mathbf{0} & -\boldsymbol{X} \boldsymbol{x} & -\boldsymbol{X} \boldsymbol{y} & -\boldsymbol{X} \\
\mathbf{0} & \mathbf{0} & \mathbf{0} & \boldsymbol{x} & \boldsymbol{y} & \mathbf{1} & -\boldsymbol{Y} \boldsymbol{x} & -\boldsymbol{Y} \boldsymbol{y} & -\boldsymbol{Y}
\end{array}\right]\left[\begin{array}{l}
m_{11} \\
m_{12} \\
m_{13} \\
m_{21} \\
m_{22} \\
m_{23} \\
m_{31} \\
m_{32} \\
m_{33}
\end{array}\right]=\left[\begin{array}{l}
\mathbf{0} \\
\mathbf{0}
\end{array}\right]
$$

En (5) $(\boldsymbol{x}, \boldsymbol{y}, \boldsymbol{X}, \boldsymbol{Y})$ son vectores $n x 1$, donde $n$ es el número de puntos utilizados para la obtención de los parámetros $m_{i j}$

\subsection{INCLUSIÓN DE NO LINEALIDADES.}

El modelo y método expuestos anteriormente son lineales y no contemplan las distorsiones producidas por la lente y el sensor PSD, por lo que la determinación de la posición no será precisa. Para minimizar el error debido a distorsión se va a añadir al sistema matricial (4), el efecto de las distorsiones radiales mediante (6) y (7):

$$
\begin{aligned}
& d_{x}=\left(x^{d}-C_{x}\right)\left(k_{1} r^{2}+\cdots+k_{n} r^{2 n}\right) \\
& d_{y}=\left(y^{d}-C_{y}\right)\left(k_{1} r^{2}+\cdots+k_{n} r^{2 n}\right)
\end{aligned}
$$

donde $\left(x^{d}, y^{d}\right)$ es la coordenada donde impacta el haz de luz sobre la superficie del sensor PSD, $r$ la distancia euclídea entre $\left(x^{d}, y^{d}\right)$ y el centro óptico del sensor, $\left(C_{x}, C_{y}\right)$ el centro óptico y $\left(k_{i=\{1, ., n\}}\right)$ los parámetros que modelan la distorsión de la lente.

Si corregimos la distorsión con 2 parámetros (dado que la focal que se utilizará no es corta) el sistema matricial queda como sigue:

$$
\left[\begin{array}{c}
s X \\
s Y \\
s
\end{array}\right]=\left[\begin{array}{lll}
m_{11} & m_{12} & m_{13} \\
m_{21} & m_{22} & m_{23} \\
m_{31} & m_{32} & m_{33}
\end{array}\right]\left[\begin{array}{c}
x+\Delta_{x} \\
y+\Delta_{y} \\
1
\end{array}\right]
$$

donde:

$$
\begin{aligned}
& \Delta_{x}=\left(x^{d}-C_{x}\right)\left(k_{1} r^{2}+k_{2} r^{4}\right) \\
& \Delta_{y}=\left(y^{d}-C_{y}\right)\left(k_{1} r^{2}+k_{2} r^{4}\right)
\end{aligned}
$$

Para obtener los parámetros que modelan el sistema (calibración), primero se obtendrán unos valores iniciales de los parámetros $m_{i j}$ mediante el método lineal y posteriormente utilizando, un método iterativo (Como por ejemplo Trust-region method) se obtendrán los parámetros finales de la matriz $\boldsymbol{M}$ y de los coeficientes de corrección de distorsión $k_{i}$, siendo la función de coste a minimizar la siguiente:

$$
f=\sum_{i=1}^{n}\left|\left(X_{i}-X_{i}^{\prime}\right)+\left(Y_{i}-Y_{i}^{\prime}\right)\right|^{2}
$$

donde $\left(X_{i}, Y_{i}\right)$ son las coordenadas de los puntos en el plano de desplazamiento, con referencia a uno de ellos, y $\left(X^{\prime}{ }_{i}, Y^{\prime}{ }_{i}\right)$ las expresiones (12) y (13).

$$
\begin{array}{r}
X_{i}^{\prime}=\frac{m_{11}\left(x+\Delta_{x}\right)+m_{12}\left(y+\Delta_{y}\right)+m_{13}}{m_{31}\left(x+\Delta_{x}\right)+m_{32}\left(y+\Delta_{y}\right)+m_{33}} \\
Y_{i}^{\prime}=\frac{m_{21}\left(x+\Delta_{x}\right)+m_{22}\left(y+\Delta_{y}\right)+m_{23}}{m_{31}\left(x+\Delta_{x}\right)+m_{32}\left(y+\Delta_{y}\right)+m_{33}}
\end{array}
$$

\section{PRUEBAS EXPERIMENTALES}

Para contrastar la validez del procedimiento sencillo y rápido de despliegue de sensores y calibración, se han llevado a cabo varias pruebas experimentales realizadas en el demostrados que el grupo Geintra dispone en la EPS de la Universidad de Alcalá. El sistema está basado en un sensor PSD de Hamamatsu Photonics C S5991-01 y emisores basado en el IRED SFH-4233 de OSRAMC que emiten señales sinusoidales de frecuencias entre 50 y $100 \mathrm{Khz}$. 
En la primera prueba se realiza una comparación de resultados de posicionamiento habiendo calibrando el sistema mediante procedimientos óptimos (que requieren de infraestructura y mucho tiempo) y con el método propuesto. La otra prueba ha consistido en el posicionamiento dinámico de dos robots, simultáneamente.

\subsection{COMPARACIÓN DE RESULTADOS CON DIFERENTES PROCESOS DE CALIBRACIÓN}

Para llevar a cabo la comparación se han realizado trayectorias circulares cuyo ground truth es conocido. Una de las medidas ha consistido en una calibración óptima del sistema determinado la posiciones mediante AoA e intersección de la recta dada por éste con el plano de movimiento de emisor (PLI) [7]; otra medida ha consistido en determinar la posición a partir de la transformación directa propuesta en este trabajo, sin corregir distorsiones (DLT), y el tercer ensayo corrigiendo distorsiones (DLT+D).

Para realizar las circunferencias se ha utilizado la plataforma mostrada en la figura 3, a la cual se ha acoplado el emisor IRED para la determinación de la posición con el sensor PSD. En las figuras 4.a, b y c se muestran los resultados de los tres ensayos realizados con circunferencias de diferentes radios, donde se comparan las posiciones obtenidas por lo diferentes métodos y sistemas. (en azul los datos medidos con el sensor PSD, utilizando la calibración optima, en verde utilizando el método DLT y en rojo el método DLT + D. Para cada una de las pruebas se han captado 2 vueltas de la circunferencia tomado 2000 muestras.

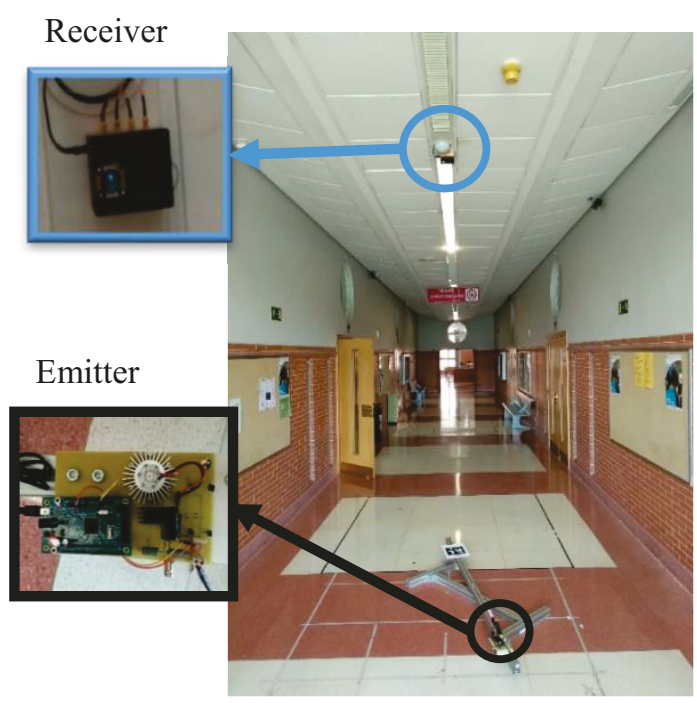

Figura 3: Escenario de pruebas
En la Tabla 1 se muestran los datos obtenidos en las pruebas, donde se comprueba que el mejor método es PLI, tanto en radio medido como en el ajuste de la circunferencia. No obstante, los resultados obtenidos mediante $\mathrm{DLT}+\mathrm{D}$ aporta resultados muy próximos, con lo que, si consideramos la infraestructura, el tiempo y sofisticación de calibración del sistema, y las precauciones a tener en el despliegue para no modificar la calibración, se justifica plenamente llevar a cabo este procedimiento mediante el método aquí propuesto.

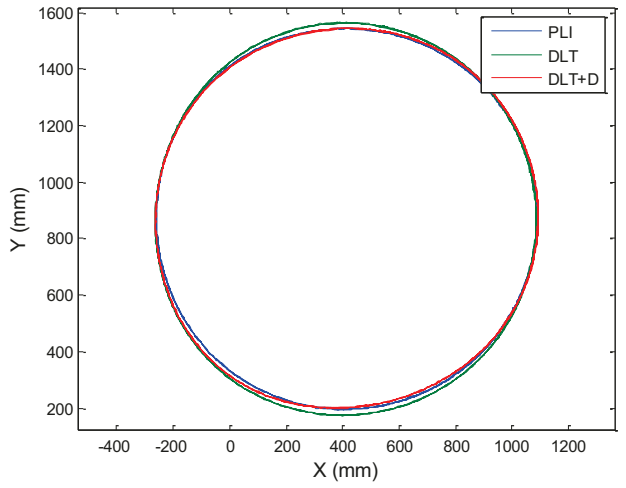

a

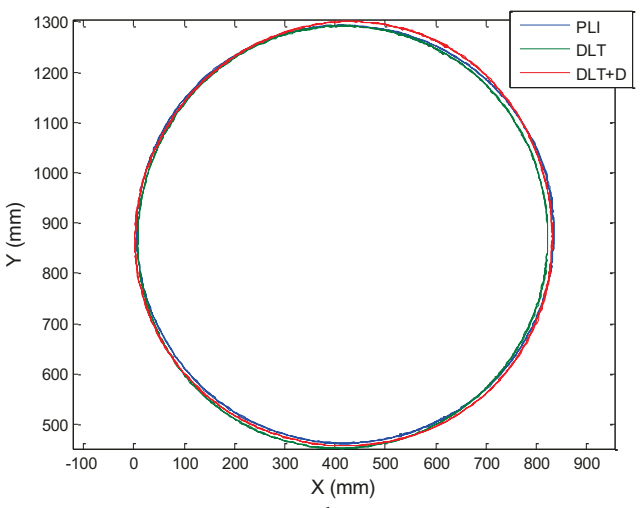

b

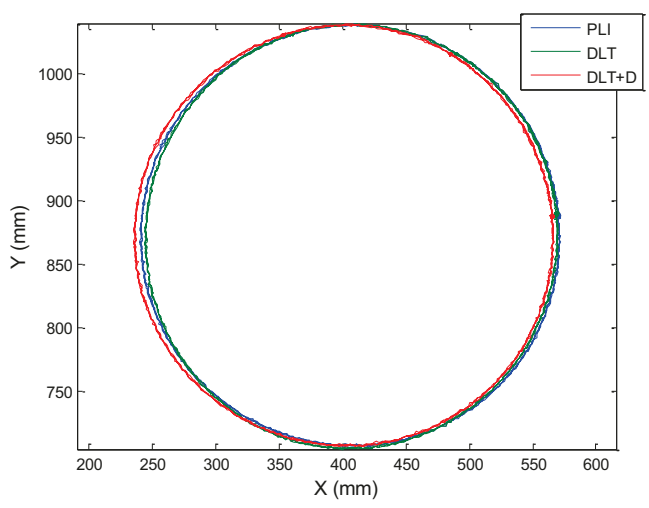

c

Figura 4: Circunferencias obtenidas por los diferentes métodos, a) Circunferencia de $650 \mathrm{~mm}$; b) Circunferencia de $400 \mathrm{~mm}$; c) Circunferencia de 160 $\mathrm{mm}$ 
Tabla 1: Resultados experimentales

\begin{tabular}{|c|c|c|c|c|c|}
\hline \multirow[b]{2}{*}{$\begin{array}{c}\text { Radio } \\
\mathrm{mm}\end{array}$} & \multirow[b]{2}{*}{ Métodos } & \multirow[b]{2}{*}{$\begin{array}{c}\text { Radio } \\
\text { medido } \\
\text { mm }\end{array}$} & \multicolumn{3}{|c|}{ Ajuste circunferencia } \\
\hline & & & $\begin{array}{l}\text { STD } \\
(\mathrm{mm})\end{array}$ & $\begin{array}{c}\text { RMSE } \\
(\mathrm{mm})\end{array}$ & $\begin{array}{l}\text { Max. } \\
\text { Error } \\
(\mathrm{mm})\end{array}$ \\
\hline \multirow{3}{*}{$650 \pm 2$} & IPR & 648.29 & 0.101 & 0.046 & 1.875 \\
\hline & DLT & 658.98 & 1.420 & 0.347 & 11.28 \\
\hline & DLT+D & 650.58 & 0.297 & 0.073 & 4.172 \\
\hline \multirow{3}{*}{$400 \pm 2$} & IPR & 398.91 & 0.112 & 0.033 & 2.289 \\
\hline & DLT & 405.66 & 0.958 & 0.238 & 6.658 \\
\hline & DLT+D & 402.93 & 0.315 & 0.075 & 3.907 \\
\hline \multirow{3}{*}{$160 \pm 2$} & IPR & 159.85 & 0.084 & 0.025 & 1.209 \\
\hline & DLT & 157.99 & 0.490 & 0.119 & 3.554 \\
\hline & DLT+D & 160.79 & 0.113 & 0.025 & 1.956 \\
\hline
\end{tabular}

\subsection{DEMOSTRACIÓN DEL SISTEMA CON DOS ROBOTS MÓVILES}

En [10] se muestra un video en el cual se mueven dos robots por el área de cobertura del sensor PSD. Para llevar a cabo una comparativa, en la prueba se obtiene la posición mediante un sistema basado en kinects y mediante el sistema basado en PSD calibrado según la propuesta DLT $+\mathrm{D}$. El control de los robots se ha realizado realimentando la posición, como se describe en [11]. Las medidas tomadas con la Kinect han sido cada $250 \mathrm{~ms}$ y las medidas con el sensor PSD cada 30 ms.

La Tabla 2 muestra los valores obtenidos en las pruebas; se muestran los resultados y los errores medios y máximos obtenidos, con los que se puede comprobar el ajuste al Ground Truth utilizando el método descrito. También se puede observar que, a pesar de ser un sistema más barato y rápido a la hora de procesar, que las cámaras, obtiene similares o mejores resultados.

Tabla 2: Resultados de pruebas dinámica

\begin{tabular}{|l|l|c|c|c|}
\hline \multirow{5}{*}{ Sistema } & \multirow{5}{*}{ Radio mm } & \multicolumn{3}{|c|}{ Ajuste circunferencia } \\
\cline { 3 - 5 } & & STD $(\mathrm{mm})$ & RMSE $(\mathrm{mm})$ & $\begin{array}{c}\text { Max. Error } \\
(\mathrm{mm})\end{array}$ \\
\hline Robot 1 \\
\hline Kinect & 659.7291 & 0.8279 & 0.2270 & 5.9258 \\
\hline \multicolumn{5}{|c|}{ Robot 2 } \\
\hline DLT+D & 674.6930 & 1.0204 & 0.1875 & 10.1186 \\
\hline Kinect & 672.9683 & 0.4219 & 0.1118 & 3.8502 \\
\hline
\end{tabular}

\section{CONCLUSIONES}

En este trabajo se ha presentado un método sencillo de calibración de un sistema IPS basado en PSD + óptica. El método simplifica enormemente el proceso de calibración de sistema además de que permite manipularlo en su despliegue sin tener que tomar precauciones especiales. Los resultados de determinación de posiciones indican que la precisión alcanzada en la calibración de parámetros es más que suficiente para que se justifique su uso, ya que posibilita el despliegue de una cantidad grande de detectores sin necesidad de disponer de sofisticadas infraestructuras de calibración ni de dedicar un excesivo tiempo. Como puede comprobarse las diferencias de resultados en espacios grandes están alrededor de los $2 \mathrm{~mm}$

\section{Agradecimientos}

En el desarrollo de este trabajo ha sido determinante el apoyo de las Redes nacionales de Excelencia de Posicionamiento y Navegación REPNIN(Ref: TEC2015-71426-REDT) y REPNIN+(Ref: TEC201790808-REDT)

\section{English summary}

\section{INDOOR POSITIONING SYSTEM BASED ON OPTICAL SIGNAL. COARSE CALIBRATION}

\begin{abstract}
Indoor Positioning Systems (IPS) usually require a calibration process to obtain precise measurements. In the case of systems with optical components, calibration may be time consuming and require a specific infrastructure. In this paper, we present a model and a calibration method for an optical sensor based on Direct Linear Transformation (DLT), which directly obtains the transformation between the sensor surface on which the signal is received and the surface of the moving agent from which signal is emitted. Our method provides results comparable to those that would be obtained with an optimal calibration, with differences of only a few mm. wide spaces.
\end{abstract}

Keywords: indoor positioning system, optical device position sensor, wireless sensing network, robotics.

\section{Referencias}

[1] Y. Zhuang, L. Hua, L. Qi, J. Yang, P. Cao, Y. Cao, Y. Wu, J. Thompson, and H. Haas, "A survey of positioning systems using visible led lights," IEEE Communications Surveys Tutorials, pp. 1-1, 2018

[2] C. Sertthin, E. Tsuji, M. Nakagawa, S. Kuwano, and K. Watanabe, "A switching estimated receiver position scheme for visible light based indoor positioning system," in 2009 4th International Symposium on Wireless Pervasive Computing, Feb 2009, pp. 1-5.

[3] R. Zhang, W. D. Zhong, K. Qian, and D. Wu, "Image sensor based visible light positioning 
system with improved positioning algorithm," IEEE Access, vol. 5, pp. 6087-6094, 2017.

[4] H. S. Kim, D. R. Kim, S. H. Yang, Y. H. Son, and S. K. Han, "An indoor visible light communication positioning system using a RFcarrier allocation technique," Journal of Lightwave Technology, vol. 31, no. 1, pp. 134144, Jan 2013.

[5] I. Fernández, M. Mazo, J. L. Lázaro, D. Pizarro, E. Santiso, P. Martín, and C. Losada, "Guidance of a mobile robot using an array of static cameras located in the environment," Autonomous Robots, vol. 23, no. 4, pp. 305-324, Nov 2007.

[6] D. Rodríguez-Navarro, J. L. Lázaro-Galilea, I. Bravo-Muñoz, A. Gardel-Vicente, and G. Tsirigotis, "Analysis and calibration of sources of electronic error in PSD sensor response," Sensors, vol. 16, no. 5, 2016.

[7] D. Rodríguez-Navarro, J. L. Lázaro-Galilea, I. Bravo-Muñoz, A. Gardel-Vicente, F. DomingoPerez, and G. Tsirigotis, "Mathematical model and calibration procedure of a PSD sensor used in local positioning systems," Sensors, vol. 16, no. 9, 2016.

[8]

https://www.hamamtsu.com/resources/pdf/s sd/psd_techinfo_e.pdf

[9] D. Rodríguez-Navarro, J. L. Lázaro-Galilea, A. De-La-Llana-Calvo, I. Bravo-Muñoz, A. GardelVicente, G. Tsirigotis, and J. Iglesias-Miguel, "Indoor positioning system based on a PSD detector, precise positioning of agents in motion using AoA techniques," Sensors, vol. 17, no. 9, 2017.

[10] [Online].

Available: https://youtu.be/TmLExaRGKuU

[11] C. Santos, M. Martínez-Rey, F. Espinosa, A. Gardel, and E. Santiso, "Event-based sensing and control for remote robot guidance: An experimental case," Sensors, vol. 17, no. 9, 2017.

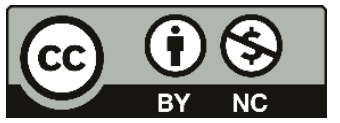

(C) 2018 by the authors. Submitted for possible open access publication under the terms and conditions of the Creative Commons Attribution CC-BY-NC $3.0 \quad$ license (https://creativecommons.org/licenses/by-nc/3.0). 